\title{
New Motion Correction Models for Automatic Identification of Renal Transplant Rejection
}

\author{
Ayman El-Baz ${ }^{1}$, Georgy Gimel'farb² ${ }^{2}$, and Mohamed A. El-Ghar ${ }^{3}$ \\ ${ }^{1}$ Bioengineering Department, University of Louisville, Louisville, KY, USA \\ ${ }^{2}$ Department of Computer Science, University of Auckland, New Zealand \\ ${ }^{3}$ Urology and Nephrology Department, University of Mansoura, Mansoura, Egypt
}

\begin{abstract}
Acute rejection is the most common reason of graft failure after kidney transplantation and early detection is crucial to survive the transplanted kidney function. In this paper, we introduce a new approach for the automatic classification of normal and acute rejection transplants from Dynamic Contrast Enhanced Magnetic Resonance Imaging (DCEMRI). The proposed algorithm consists of three main steps; the first step isolates the kidney from the surrounding anatomical structures. In the second step, new motion correction models are employed to account for both the global and local motion of the kidney due to patient moving and breathing. Finally, the perfusion curves that show the transportation of the contrast agent into the tissue are obtained from the kidney and used in the classification of normal and acute rejection transplants. In this paper, we will focus on the second and third steps and the first step is shown in detail in [1].
\end{abstract}

\section{Introduction}

In the United States, approximately 12000 renal transplants are performed annually [2], and considering the limited supply of donor organs, every effort is made to salvage the transplanted kidney [3]. However, acute rejection - the immunological response of the human immune system to the foreign kidney - is the most important cause of graft failure after renal transplantation [4, and the differential diagnosis of acute transplant dysfunction remains a difficult clinical problem.

Currently, the diagnosis of rejection is done via biopsy which has the downside effect of subjecting the patients to risks such as bleeding and infections. Moreover, the relatively small needle biopsies may lead to over or underestimation of the extent of inflammation in the entire graft [5]. Therefore, a noninvasive and repeatable technique is not only helpful but also needed in the diagnosis of acute renal rejection. In DCE-MRI, a contrast agent called Gd-DTPA is injected into the bloodstream, and as it perfuses into the organ, the kidneys are imaged rapidly and repeatedly. During the perfusion, Gd-DTPA causes a change in the relaxation times of the tissue and creates a contrast change in the images. As a result, the patterns of the contrast change gives functional information, while MRI provides good anatomical information which helps in distinguishing the

N. Ayache, S. Ourselin, A. Maeder (Eds.): MICCAI 2007, Part II, LNCS 4792, pp. 235-243, 2007.

(C) Springer-Verlag Berlin Heidelberg 2007 
diseases that affect different parts of the kidneys. However, even with an imaging technique like DCE-MRI, there are several problems such as, (i) the spatial resolution of the dynamic MR images is low due to fast scanning, (ii) the images suffer from the motion induced by the breathing patient which necessitates advanced registration techniques, and (iii) the intensity of the kidney changes non-uniformly as the contrast agent perfuses into the cortex which complicates the segmentation procedures.

To the best of our knowledge, there has been limited work on the dynamic MRI to overcome the problems of registration and segmentation. For the registration problem, Gerig et al. 6] proposed, using Hough transform, to register the edges in an image to the edges of a mask and Giele et al. 8] introduced a phase difference movement detection method to correct for kidney displacements. Both of these studies required building a mask manually by drawing the kidney contour on a 2D DCE-MRI image, followed by the registration of the time frames to this mask.

For the segmentation problem, Boykov et al. 7] presented the use of graph cuts using Markov models, where the energy is minimized depending on the manually exerted seed points. Giele et al. 8] used image subtraction to obtain a mask, and closed the possible gaps by the use of a hull function. For further segmenting the medulla and the cortex structures, repeated erosions were applied to the mask to obtain several rings; however, in such rings, the medulla structures were intermixed with the cortex structures, so a correlation study had to be applied to better classify the cortical and medullary pixels.

Following these studies, a multi-step registration approach was introduced by Sun et al. 9. Initially, the edges are aligned using an image gradient based similarity measure considering only translational motion. Once roughly aligned, a high-contrast image is subtracted from a pre-contrast image to obtain a kidney contour; which is then propagated over the other frames searching for the global registration parameters. For the segmentation of the cortex and medulla, a level sets based approach was used.

Most of these efforts used healthy transplants in the image analysis, and edge detection algorithms were sufficient. However, in the case of acute rejection patients, the uptake of the contrast agent is decreased, so edge detection fails in giving connected contours.

\section{Methods}

The objective of the proposed image analysis approach is to detect acute renal rejection from DCE-MRI images. To achieve this goal, an image analysis system consisting of three steps is proposed. These steps are: i) segmentation of kidney from DCE-MRI images, ii) correcting the motion artifacts caused by breathing and patient motion, and iii) computing the perfusion curves that show the transportation of the contrast agent into the tissue of the kidney. In this paper we will focus on the second and third steps and the first step is shown in detail in [1]. 


\section{Motion Correction Models}

In this section, we introduces two models to correct both the global and local motion of the kidney due to patient moving and breathing. The main idea of the two models is as follows: from two subsequent DCE-MRI images, we will model the visual appearance of the kidney using a Markov-Gibbs random field with pairwise interaction. Our approach is based on finding the affine transformation to register target image to the reference image by maximizing a special Gibbs energy function using a gradient descent algorithm. To get the accurate appearance model, we developed a new approach to select automatically the most important cliques that describe the visual appearance of kidney images from DCE-MRI images.

To handle local deformations, we propose a new approach based on deforming each pixel over evolving closed and equi-spaced contours (iso-contours) to closely match the prototype (reference kidney object). The evolution of the iso-contours is guided by an exponential speed function by minimizing the distances between the corresponding pixel pairs on the iso-contours on both images.

\subsection{Global Motion Model}

Basic notation. We denote $\mathcal{Q}=\{0, \ldots, Q-1\} ; \mathbf{R}=[(x, y): x=0, \ldots, X-$ $1 ; y=0, \ldots, Y-1$ ], and $\mathbf{R}_{\mathrm{p}} \subset \mathbf{R}$ a finite set of scalar image signals (e.g. gray levels), a rectangular arithmetic lattice supporting digital images $g: \mathbf{R} \rightarrow \mathcal{Q}$, and its arbitrary-shaped part occupied by the prototype (reference kidney object), respectively. A finite set $\mathcal{N}=\left\{\left(\xi_{1}, \eta_{1}\right), \ldots,\left(\xi_{n}, \eta_{n}\right)\right\}$ of $(x, y)$-coordinate offsets defines neighbors $\{((x+\xi, y+\eta),(x-\xi, y-\eta)):(\xi, \eta) \in \mathcal{N}\} \wedge \mathbf{R}_{\mathrm{p}}$ interacting with each pixel $(x, y) \in \mathbf{R}_{\mathrm{p}}$. The set $\mathcal{N}$ yields a neighborhood graph on $\mathbf{R}_{\mathrm{p}}$ to specify translation invariant pairwise interactions with $n$ families $\mathcal{C}_{\xi, \eta}$ of cliques $c_{\xi, \eta}(x, y)=((x, y),(x+\xi, y+\eta))$. Interaction strengths are given by a vector $\mathbf{V}^{\top}=\left[\mathbf{V}_{\xi, \eta}^{\top}:(\xi, \eta) \in \mathcal{N}\right]$ of potentials $\mathbf{V}_{\xi, \eta}^{\top}=\left[V_{\xi, \eta}\left(q, q^{\prime}\right):\left(q, q^{\prime}\right) \in \mathcal{Q}^{2}\right]$ depending on signal co-occurrences; here $\mathrm{T}$ indicates transposition.

Image normalization. To account for monotone (order-preserving) changes of signals (e.g. due to different illumination or sensor characteristics), the prototype and object images are equalized using the cumulative empirical probability distributions of their signals on $\mathbf{R}_{\mathrm{p}}$.

Markov Gibbs random field based appearance model. In line with a generic Markov Gibbs random field with multiple pairwise interaction, the Gibbs probability $P(g) \propto \exp (E(g))$ of an object $g$ aligned with the prototype $g^{\circ}$ on $\mathbf{R}_{\mathrm{p}}$ is specified with the Gibbs energy

$$
E(g)=\left|\mathbf{R}_{\mathbf{p}}\right| \mathbf{V}^{\top} \mathbf{F}(g)
$$

where $\mathbf{F}^{\top}(g)$ is the vector of scaled empirical probability distributions of signal co-occurrences over each clique family: $\mathbf{F}^{\top}(g)=\left[\rho_{\xi, \eta} \mathbf{F}_{\xi, \eta}^{\top}(g):(\xi, \eta) \in \mathcal{N}\right]$ 
where $\rho_{\xi, \eta}=\frac{\left|\mathcal{C}_{\xi, \eta}\right|}{\left|\mathbf{R}_{\mathrm{p}}\right|}$ is the relative size of the family and $\mathbf{F}_{\xi, \eta}(g)=\left[f_{\xi, \eta}\left(q, q^{\prime} \mid g\right)\right.$ : $\left.\left(q, q^{\prime}\right) \in \mathcal{Q}^{2}\right]^{\top}$; here, $f_{\xi, \eta}\left(q, q^{\prime} \mid g\right)=\frac{\left|\mathcal{C}_{\xi, \eta ; q, q^{\prime}}(g)\right|}{\left|\mathcal{C}_{\xi, \eta}\right|}$ are empirical probabilities of signal co-occurrences, and $\mathcal{C}_{\xi, \eta ; q, q^{\prime}}(g) \subseteq \mathcal{C}_{\xi, \eta}$ is a subfamily of the cliques $c_{\xi, \eta}(x, y)$ supporting the co-occurrence $\left(g_{x, y}=q, g_{x+\xi, y+\eta}=q^{\prime}\right)$ in $g$. The co-occurrence distributions and the Gibbs energy for the object are determined over $\mathbf{R}_{\mathrm{p}}$, i.e. within the prototype boundary after an object is affinely aligned with the prototype. To account for the affine transformation, the initial image is re-sampled to the back-projected $\mathbf{R}_{\mathrm{p}}$ by interpolation.

The appearance model consists of the neighborhood $\mathcal{N}$ and the potential $\mathbf{V}$ to be learned from the prototype.

Learning the potentials. The maximum likelihood estimator of $\mathbf{V}$ is proportional in the first approximation to the scaled centered empirical co-occurrence distributions for the prototype [1]:

$$
\mathbf{V}_{\xi, \eta}=\lambda \rho_{\xi, \eta}\left(\mathbf{F}_{\xi, \eta}\left(g^{\circ}\right)-\frac{1}{Q^{2}} \mathbf{U}\right) ;(\xi, \eta) \in \mathcal{N}
$$

where $\mathbf{U}$ is the vector with unit components. The common scaling factor $\lambda$ is also computed analytically; it is approximately equal to $Q^{2}$ if $Q \gg 1$ and $\rho_{\xi, \eta} \approx 1$ for all $(\xi, \eta) \in \mathcal{N}$. In our case it can be set to $\lambda=1$ because the registration uses only relative potential values and energies.

Learning the characteristic neighbors. To find the characteristic neighborhood set $\mathcal{N}$, the relative energies $E_{\xi, \eta}\left(g^{\circ}\right)=\rho_{\xi, \eta} \mathbf{V}_{\xi, \text { eta }}^{\top} \mathbf{F}_{\xi, \eta}\left(g^{\circ}\right)$ for the clique families, i.e. the scaled variances of the corresponding empirical co-occurrence distributions, are compared for a large number of possible candidates. Figure 1 shows a kidney prototype and its Gibbs energies $E_{\xi, \eta}\left(g^{\circ}\right)$ for 5000 clique families with the inter-pixel offsets $|\xi| \leq 50 ; 0 \leq \eta \leq 50$.

To automatically select the characteristic neighbors, let us consider an empirical probability distribution of the energies as a mixture of a large "noncharacteristic" low-energy component and a considerably smaller characteristic high-energy component: $P(E)=\pi P_{\mathrm{lo}}(E)+(1-\pi) P_{\mathrm{hi}}(E)$. Because both the components $P_{\mathrm{lo}}(E), P_{\mathrm{hi}}(E)$ can be of arbitrary shapes, we closely approximate them with linear combinations of positive and negative Gaussians. For both the approximation and the estimation of $\pi$, we use the efficient EM-based algorithms introduced in [1].

The intersection of the approximate mixture components gives an energy threshold $\theta$ for selecting the characteristic neighbors: $\mathcal{N}=\left\{(\xi, \eta): E_{\xi, \eta}\left(g^{\circ}\right) \geq\right.$ $\theta\}$ where $P_{\mathrm{hi}}(\theta) \geq P_{\mathrm{lo}}(\theta) \pi /(1-\pi)$. The above example results in the threshold $\theta=28$ producing 76 characteristic neighbors shown in Fig. 2(a),(b) together with the corresponding relative pixel-wise energies $e_{x, y}\left(g^{\circ}\right)$ over the prototype:

$$
e_{x, y}\left(g^{\circ}\right)=\sum_{(\xi, \eta) \in \mathcal{N}} V_{\xi, \eta}\left(g_{x, y}^{\circ}, g_{x+\xi, y+\eta}^{\circ}\right)
$$




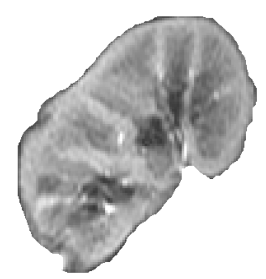

(a)

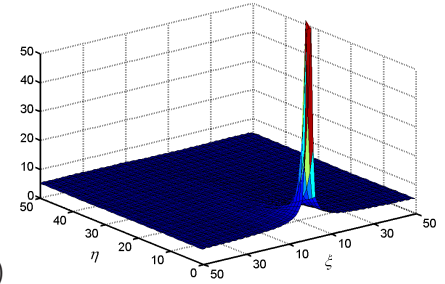

Fig. 1. Kidney image (a) and relative interaction energies (b) for the clique families in function of the offsets $(\eta, \xi)$. Note that we will repeat this step to calculate the relative energy for $n$ images in order to estimate the potentials and neighborhood system.

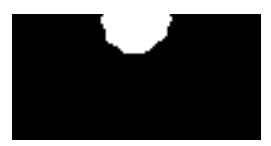

(a)

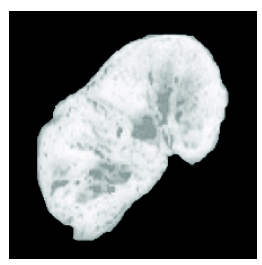

(b)

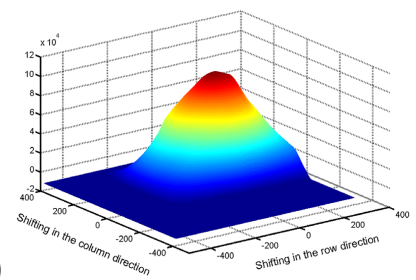

(c)

Fig. 2. (a) Most characteristic 76 neighbors among the 5000 candidates (a; in white), (b) the pixel-wise Gibbs energies for the prototype under the estimated neighborhood, and (c) Gibbs energies for translations of the object with respect to the prototype

Appearance-based registration. The object $g$ is affinely transformed to (locally) maximize its relative energy $E\left(g_{\mathbf{a}}\right)=\mathbf{V}^{\top} \mathbf{F}\left(g_{\mathbf{a}}\right)$ under the learned appearance model $[\mathcal{N}, \mathbf{V}]$. Here, $g_{\mathbf{a}}$ is the part of the object image reduced to $\mathbf{R}_{\mathrm{p}}$ by the affine transformation $\mathbf{a}=\left[a_{11}, \ldots, a_{23}\right]: x^{\prime}=a_{11} x+a_{12} y+a_{13}$; $y^{\prime}=a_{21} x+a_{22} y+a_{23}$. The initial transformation is a pure translation with $a_{11}=a_{22}=1 ; a_{12}=a_{21}=0$, ensuring the most "energetic" overlap between the object and prototype. The energy for different translations $\left(a_{13}, a_{23}\right)$ of the object relative to the prototype is shown in Fig. 2(c); the chosen initial position $\left(a_{13}^{*}, a_{23}^{*}\right)$ in Fig. 3(a) maximizes this energy. Then the gradient search for the local energy maximum closest to the initialization selects the six parameters a; Fig. 3(b) shows the final transformation aligning the prototype contour to the reference kidney. It is clear from Fig. 3(b) that the global alignment is not enough to perform perfect alignment due to the local deformation

\subsection{Local Deformation Model}

In DCE-MRI sequences, the registration problem arises because of the patient and breathing movements. To solve this problem, we propose a new approach to handle the kidney motion. The proposed approach is based on deforming the segmented kidney over evolving closed equispaced contours (i.e. iso-contours) to closely match the prototype. The evolution of the iso-contours is guided by an exponential speed function in the directions minimizing distances between 
corresponding pixel pairs on the iso-contours of both the objects to be registered. The normalized cross-correlation is used as image similarity measure which is insensitive to intensity changes (e.g. due to tissue motion in medical imagery and the contrast agent). Unlike free-form deformation approaches based on B-spline, our technique is less expensive computationally.

The first step of the proposed registration approach is to use the fast marching level set method [10 to generate the distance map inside the kidney regions as shown in Fig. 4(a)-(b). The second step is to use this distance map to generate equal space separated contours (iso-contours) as shown in Fig. 4(c)-(d). Note that the number of iso-contours depend on the accuracy and the speed required by the user. The third step of the proposed approach is to use normalized cross correlation to find the correspondence between the iso-contours. Since we start with aligned images, we limit our searching space to a small window (e.g. $10 \times 10)$ to improve the speed of the proposed approach. The final step is the evolution of the iso-contours; here, our goal is to deform the iso-contours in the first image (target image) to match the iso-contours in the second image (reference image). Before we discuss the details of the evolution algorithm, let's define the following:

$-\mathbf{b}_{\mathbf{g}_{1}}^{h}=\left[\mathbf{p}_{k}^{h}: k=1, \ldots, K\right]-K$ control points on surface $h$ on the reference data, $\mathbf{p}_{k}=\left(x_{k}, y_{k}, z_{k}\right)$ forming a circularly connected chain of line segments $\left(\mathbf{p}_{1}, \mathbf{p}_{2}\right), \ldots,\left(\mathbf{p}_{K-1}, \mathbf{p}_{K}\right),\left(\mathbf{p}_{K}, \mathbf{p}_{1}\right)$.

$-\mathbf{b}_{\mathbf{g}_{2}}^{\gamma}=\left[\mathbf{p}_{n}^{\gamma}: n=1, \ldots, N\right]-N$ control points on surface $\gamma$ on the target data, $\mathbf{p}_{n}=\left(x_{n}, y_{n}, z_{n}\right)$ forming a circularly connected chain of line segments $\left(\mathbf{p}_{1}, \mathbf{p}_{2}\right), \ldots,\left(\mathbf{p}_{N-1}, \mathbf{p}_{N}\right),\left(\mathbf{p}_{N}, \mathbf{p}_{1}\right)$.

- $S\left(\mathbf{p}_{k}^{h}, \mathbf{p}_{n}^{\gamma}\right)$ denotes the Euclidean distance between a point on surface $h$ in image $\mathbf{g}_{1}$ and its corresponding point on surface $\gamma$ in image $\mathbf{g}_{2}$,

- $S\left(\mathbf{p}_{n}^{\gamma}, \mathbf{p}_{n}^{\gamma-1}\right)$ denotes the Euclidean distance between a point on surface $\gamma$ in image $\mathbf{g}_{1}$ and its nearest point on surface $\gamma-1$ in image $\mathbf{g}_{1}$

$-\nu($.$) is the propagation speed function .$

The evolution $\mathbf{b}_{\tau} \rightarrow \mathbf{b}_{\tau+1}$ of the deformable boundary $\mathbf{b}$ in discrete time, $\tau=0,1, \ldots$, is specified by the system of difference equations $\mathbf{p}_{n, \tau+1}^{\gamma}=\mathbf{p}_{n, \tau}^{\gamma}+$ $\nu\left(\mathbf{p}_{n, \tau}^{\gamma}\right) \mathbf{u}_{n, \tau} ; n=1, \ldots, N$, where $\mathbf{u}_{n, \tau}$ is the unit vector along the ray between two correspondant points. The propagation speed function is selected so as to satisfy the following conditions: $\nu\left(\mathbf{p}_{n, \tau}^{\gamma}\right)=0$ if $S\left(\mathbf{p}_{k}^{h}, \mathbf{p}_{n, \tau}^{\gamma}\right)=0$, otherwise $\nu\left(\mathbf{p}_{n, \tau}^{\gamma}\right)=\min \left[S\left(\mathbf{p}_{k}^{h}, \mathbf{p}_{n, \tau}^{\gamma}\right), S\left(\mathbf{p}_{n, \tau}^{\gamma}, \mathbf{p}_{n, \tau}^{\gamma-1}\right), S\left(\mathbf{p}_{n, \tau}^{\gamma}, \mathbf{p}_{n, \tau}^{\gamma+1}\right)\right]$. The latter condition, known as the smoothness constraint, prevents the current point from cross-passing the closest neighbor surfaces. Note that the function $\nu\left(\mathbf{p}_{n, \tau}^{\gamma}\right)=$ $-1+\exp \left(\beta\left(\mathbf{p}_{n, \tau}^{\gamma}\right) S\left(\mathbf{p}_{k}^{h}, \mathbf{p}_{n, \tau}^{\gamma}\right)\right)$; satisfies the above conditions, where $\beta\left(\mathbf{p}_{n, \tau}^{\gamma}\right)$ is the propagation term such as, at each surface point $\beta$ is calclating from the following equation:

$$
\beta\left(\mathbf{p}_{n, \tau}^{\gamma}\right)=\frac{\ln \left(\min \left[S\left(\mathbf{p}_{k}^{h}, \mathbf{p}_{n, \tau}^{\gamma}\right), S\left(\mathbf{p}_{n, \tau}^{\gamma}, \mathbf{p}_{n, \tau}^{\gamma-1}\right), S\left(\mathbf{p}_{n, \tau}^{\gamma}, \mathbf{p}_{n, \tau}^{\gamma+1}\right)\right]+1\right)}{S\left(\mathbf{p}_{k}^{h}, \mathbf{p}_{n, \tau}^{\gamma}\right)} .
$$

Figure. 3(c) shows the result of alignment after applying the local deformation model. 


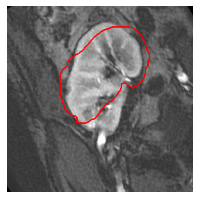

(a)
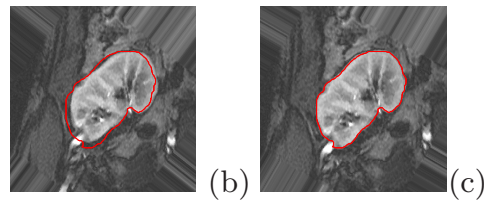

Fig. 3. (a) Initialization, (b) global alignment, and (c) local alignment
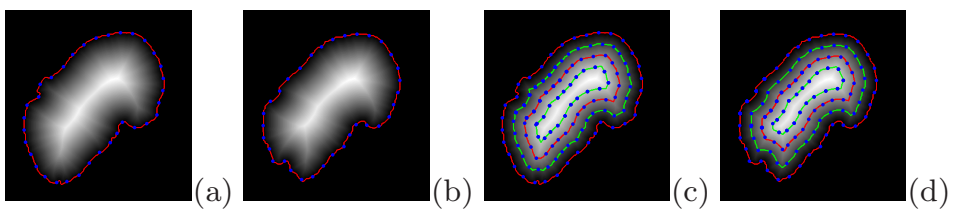

Fig. 4. The distance map of two kidneys (a, b) and the iso-contours (c, d)

\section{Results and Conclusion}

The ultimate goal of the proposed algorithms is to successfully construct a renogram (mean intensity signal curves) from the DCE-MRI sequences, showing the behavior of the kidney as the contrast agent perfuses into the transplant. In acute rejection patients, the DCE-MRI images show a delayed perfusion pattern and a reduced cortical enhancement. We tested the above algorithms on 100 patients; seven of which are shown in Figure 5(a). The normal patient shows the expected abrupt increase to the higher signal intensities and the valley with a small slope. The acute rejection patients show a delay in reaching their peak signal intensities. From these observations, we have been able to conclude that the relative peak signal intensity, time to peak signal intensity, the slope between the peak and the first minimum, and the slope between the peak and the signal measured from the last image in the sequence are the major four features in the renograms of the segmented kidney for classification. To highlight the advantage of the proposed motion correction models we drew the perfusion curves of the segmented kidneys before applying the motion correction models as shown in Fig. 5(a). It is clear from Fig. 5(a) that the measured signals are so noisy which will affect on the values of the extracted features which will be used in the classification step.

To distinguish between normal and acute rejection, we use Bayesian supervised classifier learning statistical characteristics from a training set for the normal and acute rejection. The density estimation required in the Bayes classifier is performed for each feature by using a linear combination of Gaussians (LCG) with positive and negative components. The parameters of the LCG components are estimated using a modified EM algorithm [1. In our approach, we used 50\% of the data for the training and the other $50 \%$ for testing. For testing data, the Bayes classifier succeeds to classify 47 out of 50 correctly (94\%). For the training data the Bayes classifier classifies all of them correctly, so the overall accuracy of the proposed approach is $97 \%$. The classification results before applying the 

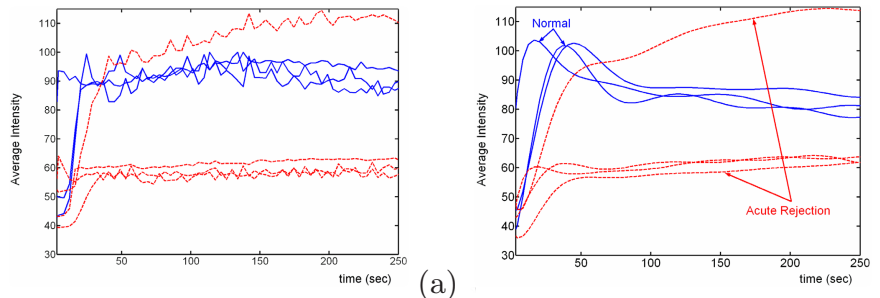

(a)

Fig. 5. (a) Perfusion curves before applying the motion correction models and (b) perfusion curves after applying the motion correction models. Note acute rejection is shown in red color and normal transplant shown in blue color.

motion correction models are as follows: 1) for testing data, the Bayes classifier succeeds to classify 29 out of 50 correctly (58\%) and 2) for the training data the Bayes classifier succeeds to classify 34 out of 50 correctly (68\%). These results highlight the advantages of our motion correction models.

In this paper we presented a framework for the detection of acute renal rejection from DCE-MRI which includes global and local registration of the kidneys and Bayes classification. Our present implementation on Matlab using Intel dual core processor, $3 \mathrm{GHz}$ each, $8 \mathrm{~GB}$ memory takes about $3.49 \mathrm{~min}$ for DCE-MRI images of size $512 \times 512$ pixels. Our future work will include testing on more patients; the results of the proposed framework are promising and might replace the current nuclear imaging tests or the invasive biopsy techniques.

\section{References}

1. El-Baz, A., Yuksel, S., Shi, H., Farag, A., El-Ghar, M., Eldiasty, T., Ghoneim, M.: 2D and $3 \mathrm{D}$ shape based segmentation using deformable models. In: Duncan, J.S., Gerig, G. (eds.) MICCAI 2005. LNCS, vol. 3750, pp. 821-829. Springer, Heidelberg (2005)

2. U.S. Department of Health and Human Services. Annual report of the U.S. scientific registry of transplant recipients and the organ procurement and transplantation network: transplant data: 1990-1999. Bureau of Health Resources Department, Richmond, VA (2000)

3. Neimatallah, M., Dong, Q., Schoenberg, S., Cho, K., Prince, M.: Magnetic resonance imaging in renal transplantation. J. Magn. Reson. Imaging 10(3), 357-368 (1999)

4. Rigg, K.M.: Renal transplantation: current status, complications and prevention. J. Antimicrob. Chem. 36(suppl.), B51-B57 (1995)

5. Yang, D., et al.: USPIO-enhanced dynamic MRI: evaluation of normal and transplanted rat kidneys. Magn. Reson. in Medicine 46, 1152-1163 (2001)

6. Gerig, G., et al.: Semiautomated ROI analysis in dynamic MRI studies: Part I: image analysis tools for automatic correction of organ displacements. IEEE Trans. Image Processing 11(2), 221-232 (1992)

7. Boykov, Y., et al.: Segmentation of dynamic N-D data sets via graph cuts using Markov models. In: Niessen, W.J., Viergever, M.A. (eds.) MICCAI 2001. LNCS, vol. 2208, pp. 1058-1066. Springer, Heidelberg (2001) 
8. Giele, E.: Computer methods for semi-automatic MR renogram determination. Ph.D. dissertation, Department of Electrical Engineering, Eindhoven University of Technology, Eindhoven (2002)

9. Sun, Y., et al.: Integrated registration of dynamic renal perfusion MR images. In: Proc. of ICIP 2004, Singapore, October, October 24-27, pp. 1923-1926 (2004)

10. Sethian, J.A.: Fast marching level set method for monotonically advancing fronts. Proc. Nat. Acad. Sci. 93, 1591-1595 (1996) 